\title{
Vehicle-to-Vehicle Connectivity Analysis for Vehicular Ad-Hoc Networks
}

\author{
Gongjun Yan*, Danda B. Rawat ${ }^{\ddagger}$ \\ * Department of Management \& Information Sciences, University of Southern Indiana, Indiana, USA. \\ Email: gyan@usi.edu ${ }^{\ddagger}$ Department of Electrical Engineering, Georgia Southern University, Statesboro, \\ GA 30458, USA. Email: db.rawat@ieee.org
}

\begin{abstract}
Vehicle-to-Vehicle (V2V) communication in Vehicular Ad hoc Networks (VANETs) is of importance in the Intelligent Transportation System (ITS) in which vehicles enlisted with wireless devices can communicate with each other. Many applications can save people's life or time on traffic such as accident alerts or congestion prediction, etc. However, network communication over VANETs is inheritedly unstable because of the high mobility of vehicles. In this paper, we analyze vehicle to vehicle wireless connectivity by using mathematic models. We consider the effect of headway distance, acceleration, association time (i.e. connection setup time), relative speed of vehicles, transmission range and message/data size in short range based V2V communications in the models. The numerical results in simulations validate the analysis.
\end{abstract}

\section{Index Terms}

Vehicle-to-vehicle communications, VANET, short range vehicular communications

\section{INTRODUCTION}

Traffic accidents and jams are causing deaths and waste of fuel and productive hours [1]. These statistics could be reduced by propagating upcoming traffic information in a timely manner using automated process in vehicular ad hoc networks (VANET) [2], [3]. Implementation of an automatic mechanism for disseminating traffic information to drivers in a timely manner can help drivers make informed decisions.

VANET is regarded as a central component for intelligent transportation system (ITS) where vehicles exchange upcoming traffic information to provide comfort to passengers, enhance traffic efficiency and safety. It is important in VANET to automate message propagation using vehicle-to-vehicle (V2V) and/or vehicle-to-roadside (V2R) with possible roadside-to-roadside ( $\mathrm{R} 2 \mathrm{R})$ communications. In V2R communications, delay is a major concern since message passes from vehicle to roadside and then roadside to destination vehicles. Where as $\mathrm{V} 2 \mathrm{~V}$ communication, delay may not be an issue but connectivity and security are major concerns as these parameters rely on neighboring participating vehicles. Safety-related applications (such as collision warning, road merging, emergency braking, etc.) short delay which can be achieved by V2V communications. Furthermore, vehicles can avoid collision with other vehicles if they talk to each other using periodic status messages wirelessely [4]. For instance, automatic cruising could be done based on the local vehicle interactions to avoid any possible collisions.

Note that there is an IEEE $802.11 \mathrm{p}$ standard for vehicular communication in $5.850 \mathrm{GHz}$ to $5.925 \mathrm{GHz}(75 \mathrm{MHz}$ RF) band as the Dedicated Short Range Communication (DSRC) to enable vehicular communication for safety and infotainment applications [5], [6]. This standard allows vehicle to transmit up to 1000 meter with $32 \mathrm{dBm}$ power.

In this paper, we investigate connectivity for short range $\mathrm{V} 2 \mathrm{~V}$ communications. We study the effect of association time, message size, transmission range and relative speed of vehicles for successful message forwarding in vehicular network. Performance is evaluated using analytical and numerical results obtained from simulations for V2V communications. Short range based wireless access technologies are also discussed for V2V communications. Note that short range based V2V communications provide VANET security from outside attackers as a byproduct [7]-[9] as road side attacker may not be able to insert malicious messages or eavesdrop the conversation.

The remainder of the paper is organized as follows: we provide a succinct survey of papers that have addressed similar issues in II. We also present the system model and formally state the problem in Section III and IV followed by an estimation of the expected value for successful message exchange in Section V. We illustrate our analytical 
results by simulation and numerical results obtained from simulations in Section VI, and present final conclusions in Section VII.

\section{RELATED WORK}

Short-range wireless communication for VANET was proposed in [10]-[14]. The work in [12] proposes NOTICE architecture where sensor belts mounted under the road every mile or so collect and transmit traffic-related messages. Vehicles are assumed to be equipped with two wireless radios (one for association and the other for message transmission). Using standard technologies association and message exchange for NOTICE architecture have been analyzed in [15]. In [14], authors have proposed so called "cat's eye" architecture in which roadside units are involved in message collecting and forwarding in vehicular network.

Panichpapiboon et al. [16] studied links and routing paths on the basis of signal strength. Kiese et al. [17] adapted received power levels and improved antenna gains to find better links. Yang et al. [18] and Kesting et al. [19] proposed using statistical and real-time density data to select wireless links in VANET. Bai et al. [20] proposed a model for path duration distribution in MANET. Based on experiments with Dynamic Source Routing (DSR), they proposed an approximate probability density function (exponential distribution) for the path duration. However, the assumption of path duration was not validated in [20].

Gruber and Hui [21] assumed link durations to be independent, exponentially distributed random variables; with this assumption, they derived the probability distributions of path duration which, not surprisingly, is exponential as well. ${ }^{1}$ However, the details of the underlying mobility model supporting such an assumption were not discussed in [21].

Pascoe et al. [22] derived the time duration of an $n$-node path. However, their mobility model only includes velocity without considering acceleration. Nekovee [23] proposed a model to determine the probability of a link in VANET under the assumptions that (1) the headway distance is constant, (2) the radio propagation model only accounts for slow fading and ignores path loss due to distance, and (3) vehicle mobility patterns are ignored. Nekovee et al. [24] assumed that car velocities are normally distributed. From this assumption, the throughput is modeled by various formulas. The path-loss is also formalized as an exponential function of velocity. This formula is also the basis of Nekovee's work [23].

Su et al. [25] proposed an analytical model for the probability density function (pdf) of link lifetime. Their model is based on several assumptions, namely that nodes are equally spaced, and that speed is normally distributed. Building on these assumptions, Sun et al. [25] computed the probability of link lifetime. However, their first assumption is not reasonable since, as widely known, inter-vehicle distance is a random variable and certainly not a constant. It is worth noting that both of these architectures are based on V2R communications and do not provide insight of association (connection setup), physical layer specifications, effect of data size, data rate, etc. Furthermore, work in [10], [11] focus on V2V based communication without considering association time, relative speed, data size, etc.

\section{V2V COMmunicATion System Model}

We assume that the vehicles are traveling on the road are equipped with computing and communication devices according to the U.S. National Highway Transportation Safety Administration (NHTSA) ruling [2]. Using those computing and communication devices, vehicles can communicate with each other using V2V based communications. We also assume that vehicles periodically broadcast their geolocations, speeds, etc. information in VANET.

Broadly speaking, there are two types of scenarios for $\mathrm{V} 2 \mathrm{~V}$ communications: $(i)$ vehicles traveling in opposite directions as shown in Fig. 1 (a) and (ii) vehicles traveling in same direction as shown in Fig. 1 (b). Note that the time interval during which two vehicles can communicate directly depends in their relative speed. It is clear that the time for information exchange using $\mathrm{V} 2 \mathrm{~V}$ communication, for a given transmission range $r$ and relative speed $v_{r e l}$, can be computed using $t=\frac{r}{v_{r e l}}$. This implies that for a given transmission range the time interval during which the vehicles can communicate decreases when their relative speed $v_{r e l}$ increases and vice versa. In other words, when the relative speed is smaller, vehicles remain within communication range for long time and they have longer time for information exchange using direct communication. It is worth noting that the relative speed of vehicles becomes

\footnotetext{
${ }^{1}$ Recall that the minimum of several independent exponential random variables is also exponential.
} 


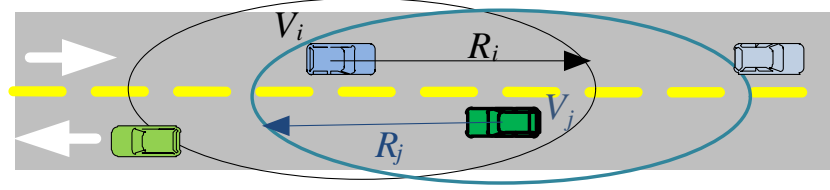

(a) Two Way Two-lane Road Section

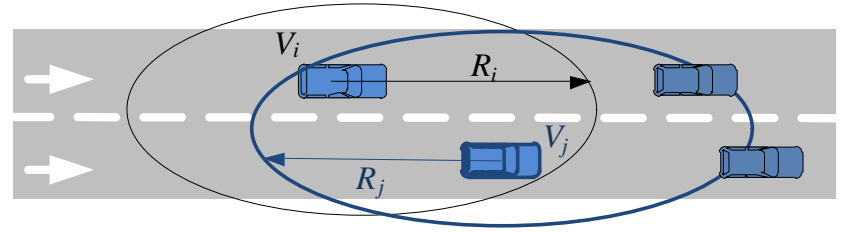

(b) One Way Two-lane Road Section

Fig. 1. Vehicular ad hoc networks with vehicles (e.g., $i$ and $j$ ) moving in their corresponding directions and with their corresponding transmission ranges (e.g., $R_{i}$ and $R_{j}$ ).

smaller (zero) when vehicles move in same direction (with equal speed). Although this situation is relative rare in urban area, it could be more normal on highways. In the case of zero relative speed V2V communication is similar to communication in static peer-to-peer network. It can provide much better reliable communication in this situation and therefore it is important for reliable V2V communication. However, when vehicles move in opposite direction, their relative speed is high since the relative speed is the sum of the speeds of two communicating vehicles and thus $\mathrm{V} 2 \mathrm{~V}$ network becomes highly dynamic ad hoc network. Note for a given transmission range and relative speed, there will be a fixed time during which vehicles remain within communication range of each other. This time must be used for both association and data exchange.

In $\mathrm{V} 2 \mathrm{~V}$ communication, wireless devices that are mounted in vehicles should be associated before exchanging the actual information or messages. That is, after successful association, vehicles are ready to exchange messages. When the vehicles travel in the same direction, because of small (or zero) relative speed, devices have sufficient time to get associated and to transmit messages. However, when the vehicles travel in opposite directions at highway speeds, they remain within communication range for a short amount of time which might not be enough to get associated and for a meaningful exchange the messages. It is well documented that different wireless technologies (such as Wi-Fi, ZigBee, Bluetooth, UWB, etc.) have different association times and data rates. These parameters play a major role in successful communication in VANET.

In the next section, we investigate the effect of association time, data rate, transmission range and relative speed of vehicles for successful V2V communications.

\section{The Probability Analysis of V2V Connectivity}

1) Path-Loss: The path-loss model [26] is a radio propagation model that predicts the signal attenuation (in $\mathrm{dB}$ ) at a distance $X$ from the transmitter. Visser et al. [27] used a patch antenna and studied the path-loss of a DSRC link. The path-loss relevant to inter-vehicle communication can be modeled by two-ray model which takes the reflection signal from the road itself into consideration. This suggests defining the path-loss in $\mathrm{dB}$ as a random variable $L(X)$ defined by writing [28]:

Lemma 1. Assuming that $X \in \log N(\mu, \sigma)$, the random variable $L(X)=40 \log X-\left(10 \log G_{t}+10 \log G_{r}+\right.$ $\left.20 \log h_{t}+20 \log h_{r}\right)$ is normally distributed.

where $^{2} G_{t}$ and $G_{r}$ are the antenna gains of the transmitter and the receiver, respectively; $h_{t}$ and $h_{r}$ are, respectively, the heights of the transmitting and receiving antennas [29]. we note that since $L(X)$ is normally distributed, its probability density function (pdf) reads $l(z) \in \frac{1}{40} N\left(\frac{\mu}{40}+b, \sigma\right)$ where $b=-\left(10 \log G_{t}+10 \log G_{r}+\right.$ $\left.20 \log h_{t}+20 \log h_{r}\right)$. To simplify the notation, we write $L(X)=Z \in a N\left(\mu_{z}, \sigma_{z}^{2}\right)$, where $a=\frac{1}{40}, \mu_{z}=a \mu+b$ and $\sigma_{z}^{2}=\sigma^{2}$.

2) On the Link Distance: We are interested in the link distance $X$, the distance between a source vehicle and a destination vehicle (i.e., between a sender and a receiver). Write $X=\sum_{i=1}^{m} X_{i}$ where the $X_{i}$ s are independent $\log$-normal random variables with a common distribution, specifically $X_{i} \in \operatorname{logN}\left(\mu_{i}, \sigma_{i}\right)$. We notice that $X_{i}$ is the headway distance between two vehicles which can be located in different lanes of road. As illustrated in Figure 2,

\footnotetext{
${ }^{2}$ Here, and in the remainder of this paper, we use $\log$ to represent $\log _{10}$ and $\ln$ to represent the natural $\operatorname{logarithm} \log { }_{e}$.
} 
$X$ represents the convolution of $m$ independent headway distances. As it turns out [30], [31], $X$ is approximately log-normal; the commonly-used Fenton-Wilkinson approximation [32] of $X$ is obtained by setting

$$
\begin{aligned}
& \sigma_{X}^{2}=\log \left[\frac{\sum e^{2 \mu_{i}+\sigma_{i}^{2}}\left(e^{\sigma_{i}^{2}}+1\right)}{\left(\sum e^{\mu_{i}+\sigma_{i}^{2} / 2}\right)^{2}}+1\right] \\
& \mu_{X}=\log \left(\sum e^{\sigma_{i}^{2}}\right)-\frac{\sigma_{Z}^{2}}{2} .
\end{aligned}
$$

To simplify the notation, we write $\sigma=\sigma_{X}$ and $\mu=\mu_{X}$ and we use the notation $X \in \operatorname{logN}(\mu, \sigma)$.

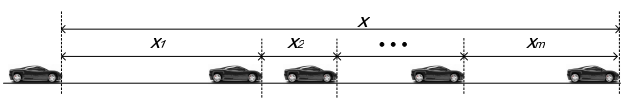

Fig. 2. Illustrating the convolution $X=X_{1}+X_{2}+\cdots+X_{m} . X_{1}, X_{2}, \cdots, X_{m}$ are independent to each other and are verified as Log-normal distribution [28]

\section{A. The Probability Distribution of Path-Loss}

A quick look at (1) reveals that the path-loss $L(X)$ is the convolution of a random variable: $Y=40 \log X$ and a constant value $-\left(10 \log G_{t}+10 \log G_{r}+20 \log h_{t}+20 \log h_{r}\right)$.

Lemma 2. Assuming that $X \in \log N(\mu, \sigma)$, the random variable $L(X)=40 \log X-\left(10 \log G_{t}+10 \log G_{r}+\right.$ $\left.20 \log h_{t}+20 \log h_{r}\right)$ is normally distributed.

The proof of the lemma is routine and is, therefore, omitted; we note that since $L(X)$ is normally distributed, its probability density function (pdf) reads $l(z) \in \frac{1}{40} N\left(\frac{\mu}{40}+b, \sigma\right)$ where $b=-\left(10 \log G_{t}+10 \log G_{r}+20 \log h_{t}+\right.$ $\left.20 \log h_{r}\right)$. To simplify the notation, we write $L(X)=Z \in a N\left(\mu_{z}, \sigma_{z}^{2}\right)$, where $a=\frac{1}{40}, \mu_{z}=a \mu+b$ and $\sigma_{z}^{2}=\sigma^{2}$.

1) The Probability Distribution of the Existence of a Link: The existence of a communication link between a source vehicle and a destination vehicle depends on the path-loss at the receiver's side. For a link between these vehicles, the path-loss between them needs to be smaller than a given threshold $P L_{t h r}$. Thus, the probability distribution $F(z)$ of the existence of a link between two vehicles separated by a distance of $z$ :

$$
\begin{aligned}
F(z) & =P\{L(X) \leq z\} \\
& =\int_{-\infty}^{z} \frac{a}{\sigma_{z} \sqrt{2 \pi}} \exp \left(-\frac{\left(t-\mu_{z}\right)^{2}}{2 \sigma_{z}^{2}}\right) d t \\
& =\frac{C_{1} a}{2}\left[1+\operatorname{erf}\left(\frac{z-\mu_{z}}{\sigma_{z} \sqrt{2}}\right)\right]
\end{aligned}
$$

where $C_{1}$ is a normalization coefficient. Since $\lim _{z \rightarrow \infty} F(z)=1$, it follows that $C_{1}=\frac{1}{a}$ and, thus, we write

$$
F(z)=\frac{1}{2}+\frac{1}{2} \operatorname{erf}\left(\frac{z-\mu_{z}}{\sigma_{z} \sqrt{2}}\right) \text {. }
$$

\section{B. The Link Duration Model}

Referring to Figure 3, assume that at time $t_{0}=0$ a link is established $\mathrm{i}$ between co-directional vehicles $i$ and $j$ with $j$ ahead of $i$. Let the random variable $X$ denote the distance separating the two vehicles at link setup time. Mindful of the $300 \mathrm{~m}$ DSRC transmission range constraint, we have

$$
0 \leq X<300 \text {. }
$$

Recall that $X$ is the convolution of $m$ independent headway distances with a common log-normal distribution [28] and that $X$ is approximately log-normal with parameters $\mu$ and $\sigma$. The assumption of log-normal distribution has been verified in the previous work [28]. We assume that the speed limit on the roadway is $v_{m}$ and that no vehicle will travel faster than $v_{m}$. For $t \geq 0$, we define $a(t)$, the acceleration of the vehicle at time $t$ as follows:

- if $a(0)=0$, then $a(t)=0$ for all $t \geq 0$; 


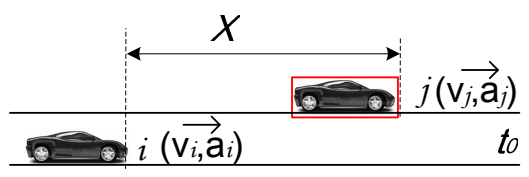

Fig. 3. Illustrating our basic scenario. Vehicle $i$ and $j$ are apart with distance $X$. Each of them has velocity and accelerate $\left(v_{i}, a_{i}\right)$ and $\left(v_{j}, a_{j}\right)$ respectively.

- if $a(0)>0$, then

$$
a(t)= \begin{cases}a(0) & \text { for } t \leq \frac{v_{m}-v(0)}{a(0)} \\ 0 & \text { otherwise }\end{cases}
$$

- if $a(0)<0$, then

$$
a(t)= \begin{cases}a(0) & \text { for } t \leq \frac{-v(0)}{a(0)} \\ 0 & \text { otherwise. }\end{cases}
$$

In other words, (4) and (5) indicate that as long as the vehicle has not reached the speed limit $v_{m}$ or has not stopped (in case $a(0)<0$ ), its acceleration remains $a(0)$. However, once the vehicle reaches the speed limit (or has stopped), its acceleration becomes 0 .

Given a generic vehicle with initial speed $v(0)$, the instantaneous speed $v(t)$ at time $t$ is defined as

$$
v(t)=v(0)+\int_{0}^{t} a(u) d u,
$$

where for all $u \in[0, t], a(u)$ is the instantaneous acceleration at time $u$ defined above.

Now, (4) and (5) and (6), combined imply that

- if $a(0)=0$, then $v(t)=v(0)$ for all $t \geq 0$;

- if $a(0)>0$, then

$$
v(t)= \begin{cases}v(0)+a(0) t & \text { for } t \leq \frac{v_{m}-v(0)}{a(0)} \\ v_{m} & \text { otherwise }\end{cases}
$$

- if $a(0)<0$, then

$$
v(t)= \begin{cases}v(0)+a(0) t & \text { for } t \leq \frac{-v(0)}{a(0)} \\ 0 & \text { otherwise }\end{cases}
$$

Similarly, with $v(x)$ defined above, the distance that our generic vehicle travels in the time interval $[0, t]$ is defined as

$$
S(t)=\int_{0}^{t} v(x) d x .
$$

We now return to our vehicles $i$ and $j$. To simplify the notation, we write $v_{i}=v_{i}(0), a_{i}=a_{i}(0)$ and $v_{j}=$ $v_{j}(0), a_{j}=a_{j}(0)$. The instantaneous speeds and accelerations $v_{i}(t)$ and $a_{i}(t)$, respectively, $v_{j}(t)$ and $a_{j}(t)$ are obtained by suitably instantiating (4), (5), (7), (8). Now, (9) guarantees that the distances traversed in the time interval $[0, t]$ by vehicles $i$ and $j$ are, respectively,

$$
S_{i}(t)=\int_{0}^{t} v_{i}(x) d x
$$

and

$$
S_{j}(t)=\int_{0}^{t} v_{j}(x) d x
$$

Assuming that at connection setup (i.e., at time 0) the distance between the two vehicles was $x$, it follows that the distance between $i$ and $j$ at time $t$ can be written as

$$
S_{j}(t)-S_{i}(t)+X
$$


It is important to note that (10) defines a signed distance: indeed, if at time $t, S_{j}(t)-S_{i}(t)+X>0$, then vehicle $j$ is ahead of $i$; otherwise, vehicle $i$ is ahead of $j$.

We find it convenient to define the indicator function $I(i, j)$ intended to capture information about which of the two vehicles is ahead when the communication link between them breaks

$$
I(i, j)= \begin{cases}1 & \text { if } S_{j}(t)-S_{i}(t)+X>0 \\ -1 & \text { otherwise }\end{cases}
$$

Given that DSRC links break at 300 meters, it follows that when the link breaks the following relation holds:

$$
S_{j}(t)-S_{i}(t)+X=300 \cdot I(i, j) .
$$

We distinguish special time moments $t_{\alpha}, t_{\beta}, t_{\gamma}$ where

- $t_{\alpha}$ is the time when two vehicles have same speed,

- $t_{\beta}$ is the time when exactly one vehicle has stopped,

- $t_{\gamma}$ is the time when both vehicles have stopped.

- $t_{\varepsilon}$ is the time when one vehicle have stopped and the other vehicle reached the speed limit $v_{m}$.

- $t_{\zeta}$ is the time when both vehicles have reached the speed limit $v_{m}$.

Due to the existence of a speed limit, we have $t_{\varepsilon}$ and $t_{\zeta}$

- $t_{\varepsilon}$ is the time when one vehicle have stopped and the other vehicle reached the speed limit $v_{m}$.

- $t_{\zeta}$ is the time when both vehicles have reached the speed limit $v_{m}$.

The reason for discussing these special time instances is that they affect in a crucial way the link duration. We now define a number of time instances that will be used in our analysis:

- provided that $\frac{v_{j}-v_{i}}{a_{j}-a_{i}}>0$, we write

$$
t_{\alpha}=\frac{v_{j}-v_{i}}{a_{j}-a_{i}}
$$

- define $t_{\beta}$ as follows

$$
t_{\beta}= \begin{cases}\frac{-v_{i}}{a_{i}} & \text { if } \frac{-v_{i}}{a_{i}}>0 \text { and } \frac{-v_{j}}{a_{j}}<0 \\ \frac{-v_{j}}{a_{j}} & \text { if } \frac{-v_{j}}{a_{j}}>0 \text { and } \frac{-v_{i}}{a_{i}}<0 \\ \min \left\{\frac{-v_{i}}{a_{i}}, \frac{-v_{j}}{a_{j}}\right\} & \text { if } \frac{-v_{i}}{a_{i}}>0 \text { and } \frac{-v_{j}}{a_{j}}>0 \\ \text { undefined } & \text { otherwise. }\end{cases}
$$

- similarly, define $t_{\gamma}$ as follows

$$
t_{\gamma}= \begin{cases}\max \left\{\frac{-v_{i}}{a_{i}}, \frac{-v_{j}}{a_{j}}\right\} & \text { if } \frac{-v_{i}}{a_{i}}>0 \text { and } \frac{-v_{j}}{a_{j}}>0 \\ \text { undefined } & \text { otherwise. }\end{cases}
$$

- define $t_{\varepsilon}$ as follows

$$
t_{\varepsilon}= \begin{cases}\frac{v_{m}-v_{i}}{a_{i}} & \text { if } \frac{-v_{i}}{a_{i}}>0 \text { and } \frac{v_{m}-v_{j}}{a_{j}}<0 \\ \frac{v_{m}-v_{j}}{a_{j}} & \text { if } \frac{-v_{j}}{a_{j}}>0 \text { and } \frac{v_{m}-v_{i}}{a_{i}}<0 \\ \min \left\{\frac{v_{m}-v_{i}}{a_{i}}, \frac{v_{m}-v_{j}}{a_{j}}\right\} & \text { if } \frac{v_{m}-v_{i}}{a_{i}}>0 \text { and } \frac{v_{m}-v_{j}}{a_{j}}>0 \\ \text { undefined } & \text { otherwise }\end{cases}
$$

- define $t_{\zeta}$ as follows

$$
t_{\zeta}= \begin{cases}\max \left\{\frac{v_{m}-v_{i}}{a_{i}}, \frac{v_{m}-v_{j}}{a_{j}}\right\} & \text { if } \frac{v_{m}-v_{i}}{a_{i}}>0 \text { and } \frac{v_{m}-v_{j}}{a_{j}}>0 \\ \text { undefined } & \text { otherwise }\end{cases}
$$

It is important to note that $\left\{t_{\alpha} \leq t_{\beta} \leq t_{\gamma}\right\}$ and $\left\{t_{\varepsilon}, t_{\zeta}\right\}$ only depend on the speeds and acceleration of the two vehicles at connection setup time and on the value of the speed limit $v_{m}$.

\section{Deriving the Link Duration}

Because of obvious similarities, we only discuss the scenario that both vehicle move in the same direction and have positive accelerations. Sooner or later they will reach the speed limit and will, thereafter, cruise at the maximum speed. 
1) $0 \leq t \leq t_{\alpha}$ : In this simulation, when the link breaks, vehicle $j$ must be ahead of $i$ and, thus, $I(i, j)=1$. Recalling that in DSRC the link breaks when the distance between $i$ and $j$ is 300 meters, we can write (11) as

$$
S_{j}(t)-S_{i}(t)+X=300 .
$$

On the other hand, by (9), $S_{j}(t)-S_{i}(t)=\frac{1}{2} a_{r} t^{2}+v_{r} t$. where $a_{r}=a_{j}-a_{i}, v_{r}=v_{j}-v_{i}$. All that remains, is to substitute $S_{j}(t)-S_{i}(t)$ in (12) and to solve for $t$. Since $t<t_{\alpha}$, we obtain

$$
t=\frac{-v_{r}+\sqrt{v_{r}^{2}+2 a_{r}(300-X)}}{a_{r}} .
$$

2) $t_{\alpha}<t \leq t_{\varepsilon}$ : In this case, when the link breaks vehicle $i$ must be ahead of vehicle $j$; thus $I(i, j)=-1$ and by (11) we can write

$$
S_{j}(t)-S_{i}(t)+X=-300 .
$$

We know that $S_{j}(t)-S_{i}(t)=\frac{1}{2} a_{r} t^{2}+v_{r} t$. By substituting the value of $S_{j}(t)-S_{i}(t)$ in (IV-C2) and by solving for $t$ we obtain

$$
t=\frac{-v_{r}-\sqrt{v_{r}^{2}-2 a_{r}(300+X)}}{a_{r}} .
$$

3) $t_{\varepsilon}<t \leq t_{\zeta}$ : In this case, when the link breaks, vehicle $i$ must be ahead of vehicle $j$ and so $I(i, j)=-1$. Thus, we can write

$$
S_{j}(t)-S_{i}(t)+X=-300 .
$$

Observe that by (9), $S_{j}(t)=\frac{1}{2} a_{j} t^{2}+v_{j} t$ and $S_{i}(t)=v_{m} t-\frac{v_{m}-v_{i}}{2} t_{\varepsilon}$. After substituting the value of $S_{j}(t)-S_{i}(t)$ in (IV-C3) and after solving for $t$ we obtain

$$
t=\frac{-\left(v_{j}-v_{m}\right)-\sqrt{\left(v_{j}-v_{m}\right)^{2}-2 a_{j}\left(300+x+\frac{v_{m}-v_{i}}{2} t_{\varepsilon}\right)}}{a_{j}} .
$$

4) $t_{\zeta}<t$ : In this case, the link will not break because the two vehicles move at the same speed (alternatively, the link breaks at $+\infty$ ).

The scenario shows that vehicle $i$ catches up with vehicle $j$, passes $j$ and, finally, breaks the link with vehicle $j$. Experience tells us that this is a very frequent occurrence in highway traffic.

\section{The Distribution Function of Link Duration}

The duration of a link is the lifetime of an established communication link between two vehicles. The main goal of this section is to derive analytical expressions for the probability distribution and density function of the duration of a link.

With the preamble of the previous section out of the way, we are now ready to state and prove the following important result. The proofs of Lemmas can be referred in Appendices.

Lemma 3. Assuming $X \in \log N(\mu, \sigma)$, the random variable $T=\sqrt{a X+b}+c$ is log-normally distributed, where $a, b, c \in \mathbb{R}, a, b, c \neq 0$ and $a X+b \geq 0$.

Lemma 4. Assuming that $X$ is log-normal with parameters $\mu$ and $\sigma$, the random variable $T=a X+b$ is lognormally distributed, where $a, b, c \in \mathbb{R}$ and $a, b, c \neq 0$.

Lemma 5. Suppose that the communication link between two vehicles $i$ and $j$ breaks at time $t$. The link duration time is either a linear function of $X$ or a square root function of $X$.

Then we come up with Theorem 6 as below. The proof can be referred in appendices.

Theorem 6. The duration $T$ of the link between vehicles $i$ and $j$ is log-normally distributed. 
TABLE I

Comparison of The Bluetooth, ZigBee, And Wi-Fi Protocols

\begin{tabular}{|l|l|l|l|}
\hline Standard & Bluetooth & ZigBee & WiFi \\
\hline IEEE spec. & 802.15 .1 & 802.15 .4 & $802.11 \mathrm{a} / \mathrm{b} / \mathrm{g}$ \\
\hline Frequency band & $2.4 \mathrm{GHz}$ & $868 / 915 \mathrm{MHz} ; 2.4 \mathrm{GHz}$ & $2.4 \mathrm{GHz} ; 5 \mathrm{GHz}$ \\
\hline Max signal rate & $1 \mathrm{Mb} / \mathrm{s}$ & $250 \mathrm{~Kb} / \mathrm{s}$ & $54 \mathrm{Mb} / \mathrm{s}$ \\
\hline Nominal range & $10 \mathrm{~m}$ & $10-100 \mathrm{~m}$ & $100 \mathrm{~m}$ \\
\hline Nominal TX power & $0-10 \mathrm{dBm}$ & $(-25)-0 \mathrm{dBm}$ & $15-20 \mathrm{dBm}$ \\
\hline Number of RF channels & 79 & $1 / 10 ; 16$ & $14(2.4 \mathrm{GHz})$ \\
\hline Channel bandwidth & $1 \mathrm{MHz}$ & $0.3 / 0.6 \mathrm{MHz} ; 2 \mathrm{MHz}$ & $22 \mathrm{MHz}$ \\
\hline Modulation type & GFSK & BPSK $(+$ ASK), O-QPSK & BPSK, QPSK, COFDM, CCK, M-QAM \\
\hline Spreading & FHSS & DSSS & DSSS, CCK, OFDM \\
\hline Coexistence mechanism & Adaptive freq. hopping & Dynamic freq. selection & Dynamic freq. selection, power control (802.11h) \\
\hline Basic cell & Piconet & Star & BSS \\
\hline Extension of the basic cell & Scatternet & Cluster tree, Mesh & ESS \\
\hline Max number of cell nodes & 8 & $>65000$ & 2007 \\
\hline Encryption & EQ stream cipher & AES block cipher $(\mathrm{CTR}, \mathrm{CTR}$ mode) & RC4 stream cipher (WEP), AES block cipher \\
\hline Authentication & Shared secret & CBC-MAC (ext. of CCM) & WPA2 (802.11i) \\
\hline Data protection & $16-b i t$ CRC & $16-b i t$ CRC & $32-$ bit CRC \\
\hline Approx. Association time & 45 ms & 650 ms & $4 \mathrm{~s}$ \\
\hline
\end{tabular}

Let $\Phi$ be a set of all real combination of $v_{i}, v_{j}, a_{i}, a_{j}$ on roads and $\phi$ be the size of $\Phi$. Let $P_{k}$ be the probability of the case $k \in \Phi$ and $T_{k}$ be the link duration time of case $k$. By the law of total expectation, we can obtain the overall expected duration of a link $E[$ link $]$,

$$
E[\text { link }]=\sum_{k=1}^{\phi} P_{k} T_{k} .
$$

Theoretically, $E[$ link $]$ can be computed by (13). However, to the best of our knowledge, there are no analytical results or field-test data on $P_{k}$ and $T_{k}$ in literature. We will leave the computation of the expected link duration for future investigations.

\section{Analysis for SucCessful Data Exchange}

Let $A, T$ and $L$ be, respectively, association time ${ }^{3}$, data exchange time (time left after successful association) and total available time for given transmission range in $\mathrm{V} 2 \mathrm{~V}$ communication. For successful association and data exchange, $A+T \leq L$ condition must be satisfied. Comparison of ZigBee, Bluetooth and Wi-Fi technology is presented in Table 1. Probability of successful association and data exchange between vehicles can be expressed as $P_{s}=\operatorname{Pr}\{A+T \leq L\}$. Then $1-P_{s}$ gives the probability of unsuccessful data exchange which represents, in our case, partial or no messages being exchanged between vehicles. Value of total available time for V2V communication can be computed as

$$
L= \begin{cases}\frac{D_{i, j}}{v_{r e l}}, & D_{i, j} \leq \min \left\{R_{i}, R_{j}\right\} \quad \forall i, j \\ 0, & \text { Otherwise }\end{cases}
$$

where $D_{i, j}$ is the distance between vehicles $i$ and $j$. Assuming that the two vehicles are located at $\left(x_{i}, y_{i}\right)$ and $\left(x_{j}, y_{j}\right)$, we can compute $D_{i, j}$ as

$$
D_{i, j}=\sqrt{\left(x_{i}-x_{j}\right)^{2}+\left(y_{i}-y_{j}\right)^{2}}
$$

Obviously, for the vehicles to be able to communicate, we must have $D_{i, j} \leq \min \left\{R_{i}, R_{j}\right\}$. From equation (14), it is clear that when $v_{r e l} \rightarrow 0$, the value of $L \rightarrow \infty$. This implies that when the vehicles move at constant speed in the same direction while exchanging their information, they will have very long time (theoretically $\infty$ ) to get associated and exchange the messages.

Based on the analysis above, we present the algorithm for time calculation as Algorithm 1:

\footnotetext{
${ }^{3}$ Note that the association time depends on technologies such as typical association time for ZigBee is about 30 milliseconds, Wi-Fi is about 600 milliseconds and so on [33].
} 


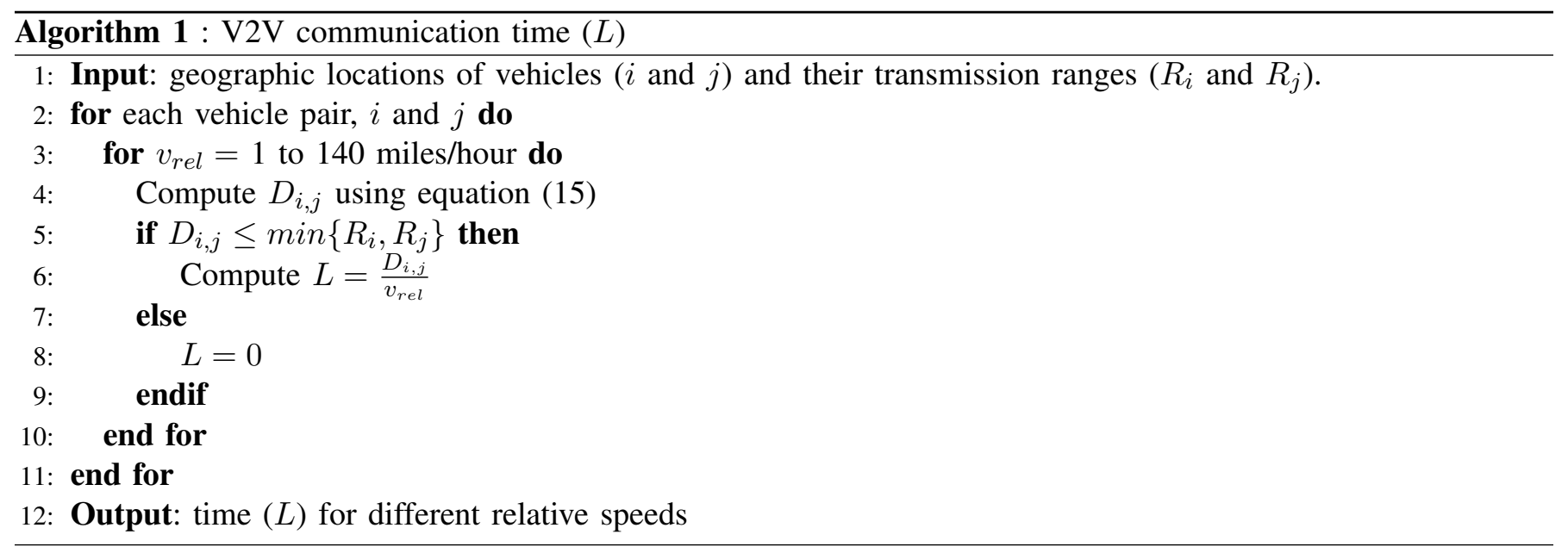

Once we have the total time for V2V communication, we can compute the time for message exchange (that is the time left after successful association of devices) and amount of data that could be exchanged between vehicles using Algorithm 2.

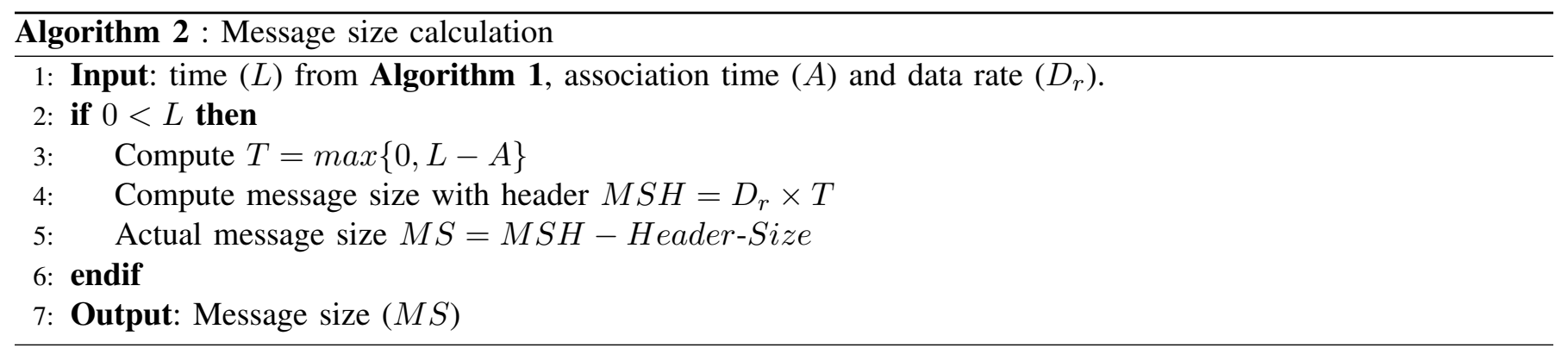

Furthermore, without transmitting a complete message, the message would have no meaning in VANETs. Note that when partial message is exchanged in $\mathrm{V} 2 \mathrm{~V}$ communication, receiving vehicle may not be able to understand the actual semantic/meaning of the message unless it gets a copy of the message from other vehicles. Thus, we consider that the complete message of size $S$ has to be transmitted with given data rate $D_{r}$ for successful V2V communication. The time $T$ needed to transmit given message of size $S$ is given by

$$
T=\frac{S}{D_{r}}
$$

Because of dynamic nature of VANET topology, it is important to estimate the time (available for data exchange) after successful association of wireless devices. Note that this value depends on various parameters such as relative speed of vehicle, transmission range, association time of technology being used, etc.

Estimation of expected time for data exchange: We assume that communication range overlap between vehicles is random process and it does not depend on the time and range overlap of the previous vehicles. We consider distribution of vehicles being within a communication range of each other as Poisson distribution with parameter $\beta$ [34, Ch. 8] to analyze how association time can influence the data exchange in V2V communications. Note that $L \geq 0$ is the random variable representing the total time/duration available for device association and data exchange between vehicles for $\mathrm{V} 2 \mathrm{~V}$ communications which follows the Poisson distribution with parameter $\beta$. Thus the cumulative distribution function (cdf) for a random variable $L$ is defined as [34]

$$
F_{\boldsymbol{L}}(L)=1-e^{-\beta L}, \quad L \geq 0
$$

Then we can find probability distribution function (pdf) as

$$
f_{L}(L)= \begin{cases}\beta e^{-\beta L}, & L \geq 0 \\ 0, & \text { Otherwise }\end{cases}
$$


Using total time available $L$ and association time $A$, we can write time for data exchange (after connection setup) as

$$
\boldsymbol{T}=[\boldsymbol{L}-\boldsymbol{A}]^{+}
$$

Where $[b]^{+}=\max \{0, b\}$. Note that $T$ is also a random variable and should be positive to be able to transmit messages using $\mathrm{V} 2 \mathrm{~V}$ communication. Then we can write probability of successful message exchange for given time $T$ as

$$
\begin{aligned}
F_{\boldsymbol{T}}(T)=P\{\boldsymbol{T} \leq T\} & =P\{\boldsymbol{L}-A \leq T\} \\
& =P\{\boldsymbol{L} \leq T+A\} \\
& =F_{\boldsymbol{L}}(T+A)
\end{aligned}
$$

Now, we can write cdf as

$$
F_{\boldsymbol{T}}(T)=1-e^{-(T+A) \beta}
$$

and then pdf as

$$
f_{\boldsymbol{T}}(T)=\beta e^{-(T+A) \beta}
$$

Then the expected value of time $T$ can be computed as

$$
\begin{aligned}
\bar{T}=E[\mathbf{T}] & =\int_{0}^{\infty} T \beta e^{-(T+A) \beta} d x \\
& =\int_{0}^{\infty} T \beta e^{-T \beta} e^{-A \beta} d x \\
& =\frac{1}{\beta} e^{-A \beta}
\end{aligned}
$$

Note that (23) gives the expected value of usable time as a function of successful association time when we consider $T$ as a random variable and there is no boundary in the transmission range. In our case, transmission range is given by (15). Considering factors such as distance between vehicles, data rate, data size, etc, we compute ratio of time, $P_{r}$, as

$$
P_{r}=\min \left\{1,\left[\frac{\left(\frac{D_{i, j}}{v_{r e l}}\right)}{\left(A+\frac{S}{D_{r}}\right)}\right]\right\}
$$

It is worth noting that ratio $P_{r}$ values is bounded by $[0,1]$ and its value is 1 when relative speed is zero (this happens when two vehicles travel with equal speed in the same direction). Then, the expected time $\bar{T}$ becomes

$$
\begin{aligned}
\bar{T} & =\frac{1}{\beta} e^{-A \beta} \times P_{r} \\
& =\frac{1}{\beta} e^{-A \beta} \times \min \left\{1,\left[\frac{\left(\frac{D_{i, j}}{v_{\text {rel }}}\right)}{\left(A+\frac{S}{D_{r}}\right)}\right]\right\}
\end{aligned}
$$

Note that the expected time $\bar{T}$ for data exchange for given association time and data rate of the technology, and overlapping of transmission range and relative speed of communicating vehicles. From (25), it follows that when relative speed is zero, $P_{r}$ becomes 1 and the expected value $\bar{T}$ depends only on association time $A$ for a given $\beta$.

Using (14) and (25), the probability of successful data exchange is expressed as

$$
P_{s}=\operatorname{Pr}\{A+\bar{T} \leq L\}
$$

This probability depends on association time and data rate of a given technology, relative speed of communicating vehicles and size of the message to be transmitted. 

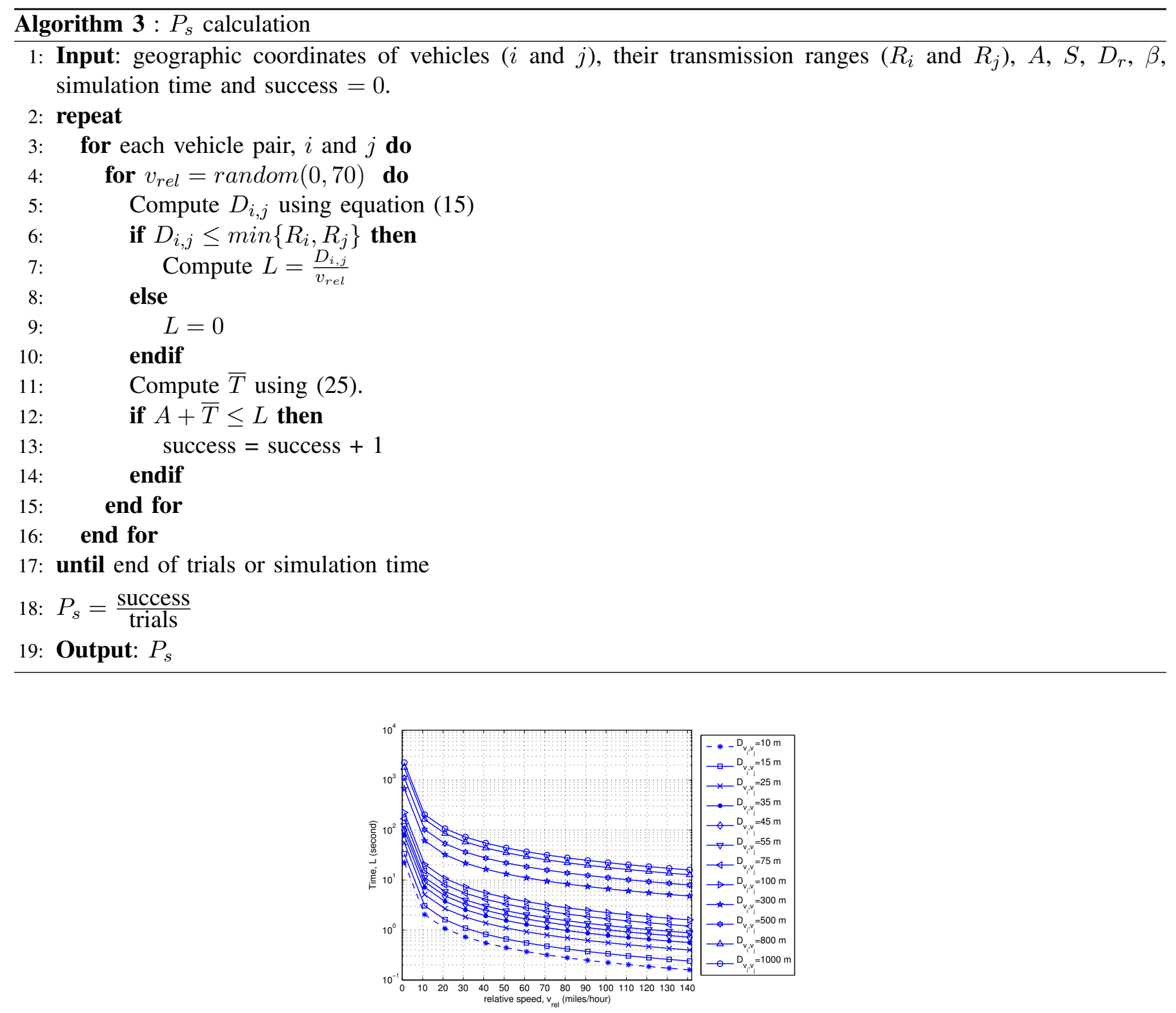

Fig. 4. Upper limit of total communication time (L) for vehicles $v_{i}$ and $v_{j}$ for different relative speeds (starting from 1 miles/hour through 140 miles/hour) and communication ranges of $D_{v_{i}, v_{j}}$ (starting from 10 meters through 1000 Meters - DSRC recommended maximum range).

\section{Vi. Simulation AND Results}

We considered that the overlap of communication range between vehicles is Poisson distribution process with the parameter $\beta=1$ vehicle/second, and overlap in range does not depend on the time and range overlap of the previous vehicles. Relative speed of vehicles varied from 0 mile/hour to 140 miles/hour.

In the first experiment, using Algorithm 1, we plotted the variation in available time for given transmission range (varying from 10 meter through 1000 meter) and different relative speeds of vehicles (varying from 1 mile/hour through 140 miles/hour ${ }^{4}$ ) as shown in Fig. 4. As expected for a given transmission range, when relative speed of vehicles increases, available time for communication decreases. For a given relative speed (e.g. 40 miles/hour), time increases when transmission range increases. Observe that the sum of association time and the time needed for message/data exchange cannot exceed the line in Fig. 4 for a given transmission range and relative speed.

\footnotetext{
${ }^{4}$ Posted speed of the US roads/highways is not greater than 70 miles/hour. Thus the relative speed of 140 miles/hour is for vehicles moving with 70 miles/hour in opposite direction.
} 


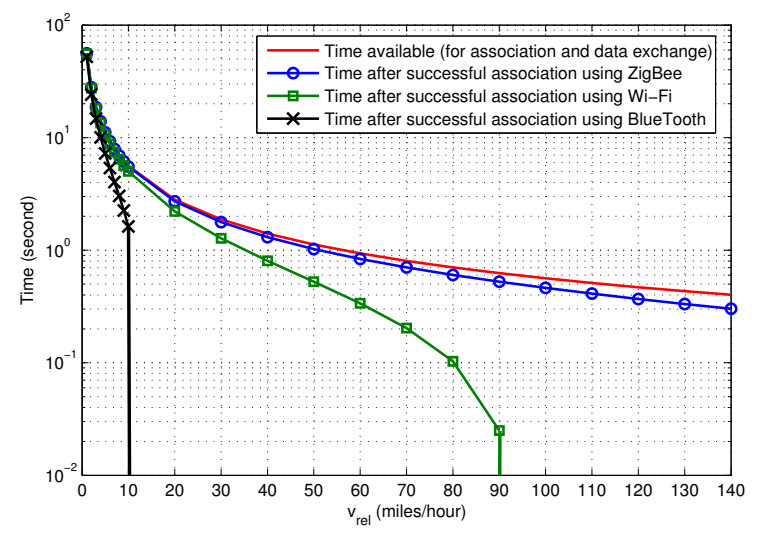

Fig. 5. Total time and time left after successful association between vehicles $v_{i}$ and $v_{j}$ for different relative speeds and communication range equal to $D_{v_{i}, v_{j}}=25$ meter.

In the second experiment, we have considered three scenarios where vehicles are assumed to be equipped with Bluetooth, Wi-Fi, ZigBee/xBee devices ${ }^{5}$ for short range $\mathrm{V} 2 \mathrm{~V}$ communications. We calculated the total available time for given transmission ranges and relative speeds of vehicles and plotted both total time and time remaining after successful association in Fig 5. Note that Bluetooth, Wi-Fi and ZigBee devices take, respectively, about 4 seconds, 600 milliseconds and 30 milliseconds for association or connection setup [33]. After successful association, devices exchange actual information. Fig 5shows that the vehicles with Bluetooth devices had no time left when their relative speed is greater than 10 miles/hour since Bluetooth devices could not be associated or they spent all of the available time for association. As a result, vehicles could not exchange messages when they traveled with a relative speed greater than 10 miles/hour as shown in Fig 6 (a). Note that the size of the message transmitted is about 4000 Kilobytes when relative speed is about 10 miles/hour.

Next, we assumed that each vehicle was equipped with a Wi-Fi device. When the relative speed was not greater than 90 miles/hour, Wi-Fi devices exchanged messages using V2V communications as shown in Fig 6 (b). For a relative speed of 90 miles/hour, vehicles exchanged 6 Kilobytes of messages. Note that the size of a typical one-page message with 568 words is about 5 Kilobytes.

Finally, we assumed that individual vehicles were equipped with ZigBee devices. ZigBee devices were able to transmit messages of size 90 Kilobytes even when their relative speed was 140 miles/hour.

It is noted that for a given transmission range of 25 meter and relative speed not greater than 90 miles/hour, Wi-Fi devices could be used for successful V2V communications. Similarly, Bluetooth devices could be used only when relative speed is not greater than 10 miles/hour. However, ZigBee devices could be used to exchange messages even when relative speed is about 140 miles/hour or even higher.

To see the effect in message exchange, we then changed the transmission range from 25 meter to 10 meter for all three cases and plotted the data exchanged for given relative speeds as shown in Fig 6. We noted that vehicles could not exchange data when they move with relative speed higher than 5 miles/hour and 30 miles/hour using Bluetooth and Wi-Fi devices respectively. Furthermore, message size decreased significantly when we decreased the transmission range to 10 meter in all cases as shown in Fig 6 (a). (b) and (c). It is noted that tradeoff between transmission range and message should be considered.

Finally, we computed probability of successful data exchange in V2V communication using Algorithm 3 and plotted it for given message size of 5 Kilobytes (typical size of one page message of 568 words) using different data rates and association time by averaging 1000 trials. Relative speeds of vehicles were generated randomly in the range $70 \pm 70$ miles/hour. This represents both low and high relative speeds ranging from 0 miles/hour to 140 miles/hour. From Fig 7, note that as expected, when association time increases, probability of successful data exchange decreases. It is worth noting that for given transmission range and relative speed, there is a fixed time during where vehicles remain within communication range of each other. The overlap time is used for both

\footnotetext{
${ }^{5}$ Note that to make typical wireless technologies such as Bluetooth, Wi-Fi, ZigBee/xBee, etc. suitable for V2V communications, specifications such as transmission range/power could have to be changed to meet the needs for $\mathrm{V} 2 \mathrm{~V}$ communications.
} 


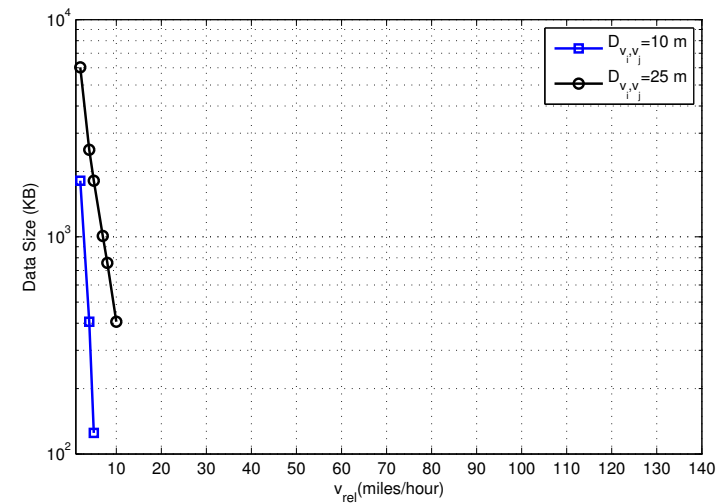

(a) Bluetooth Technology

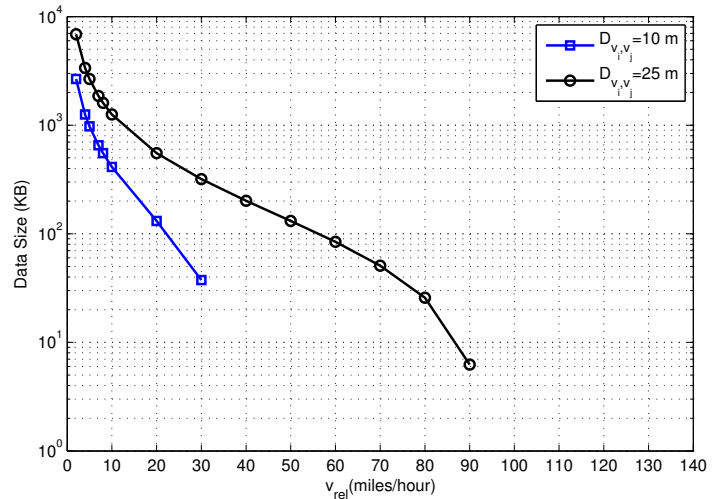

(b) Wi-Fi Technology

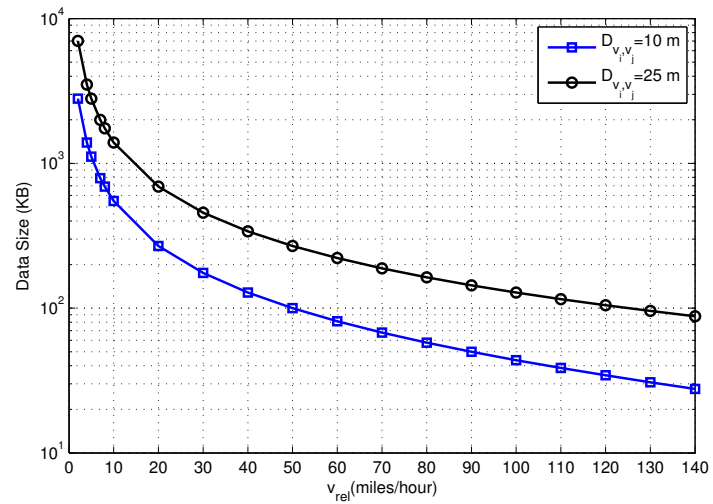

(c) ZigBee/XBee Technology

Fig. 6. Upper bound of message size that could be transmitted with a given data rate of $250 \mathrm{Kbps}$ for different relative speed of vehicles and communication range $D_{v_{i}, v_{j}} \in\{10,25\}$ meter.

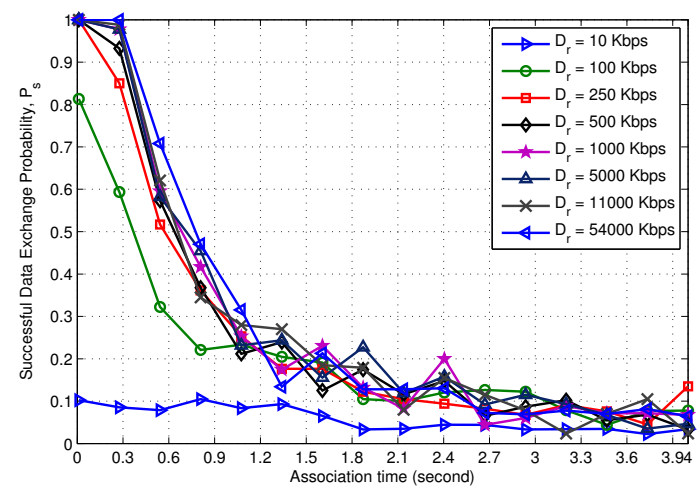

Fig. 7. Effect of Association Time for given message size (e.g. the size of a typical one page message with 568 words is about 5 Kilobytes) and data rate on successful data exchange probability for $\mathrm{V} 2 \mathrm{~V}$ based communication when the communication range equal to $D_{v_{i}, v_{j}}=25$ meter.

association and data exchange, when association time increases, time left for data exchange decreases resulting in unsuccessful data exchange. Again, note that unsuccessful data exchange in our case is partial or no information is exchanged at all. Furthermore, to exchange typical one page message (i.e., 5 Kilobytes), there is no significant difference in probability $P_{s}$ value when data rate is higher than or equal to $100 \mathrm{Kbps}$.

We conclude that $\mathrm{V} 2 \mathrm{~V}$ communication requires fast association between wireless devices for successful message exchange between communicating vehicles to forward messages.

We varied the speed (both $v_{i}$ and $v_{j}$ ) in cases I and II and recorded the link duration. Figure 8 illustrated the 
results we obtained. We assume that the link duration 100 seconds represent infinite link duration. Figure 8(a) shows that larger speed of vehicle $i$ will cause longer link duration and larger speed of vehicle $j$ will cause shorter link duration because both $i$ and $j$ are accelerating in case I. When $v_{i}$ increases, it increases the likelihood of reaching $v_{m}$. Therefore both $i$ and $i$ reach the speed limit $v_{m}$ before the link breaks. Tus, in this case, the link tends to be stable. As we expected, the simulation results match well the theoretically-predicted values, as shown in Figure 8(b). The higher speed of vehicle $j$ will cause a shorter link duration, and the higher speed of vehicle $i$ will cause a longer link duration. If vehicle $j$ has higher speed, the relative speed between $j$ and $i$, i.e. $v_{j}-v_{i}$ will be higher. Therefore, the link duration will be shorter.

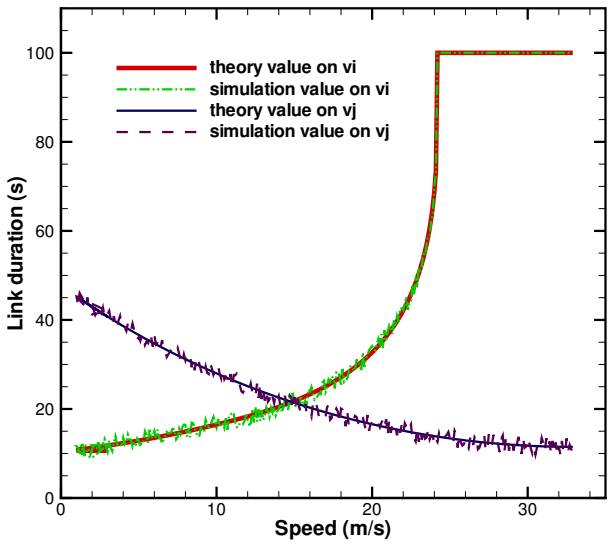

(a) Speed impact in case I.

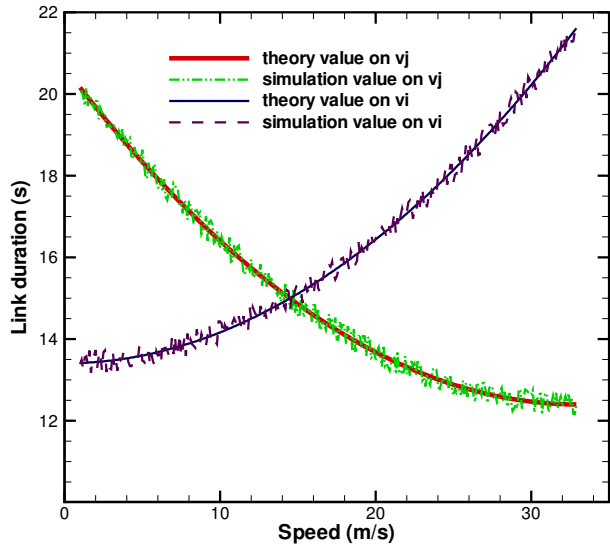

(b) Speed impact in case II.

Fig. 8. Speed has significant impact on link duration.

\section{CONCLUSION}

In this paper, we presented the analysis for information exchange success rate and probability of constructing communication link using V2V communication for vehicular network. Both analysis models considered mobility factors such as headway distance, relative speed, acceleration, and time. As a conclusion remark, some common wireless devices can be used to exchange the information using V2V communication. For a given transmission range, ZigBee devices could be used when relative speed is high (e.g. 140 miles/hour), Wi-Fi devices could be used when relative speed is not greater than 90 miles/hour. Bluetooth could be used when relative speed is not greater than 10 miles/hour.

In many applications especially emergency related applications, $\mathrm{V} 2 \mathrm{~V}$ communication are required to provide fast association and low communication latency between communicating vehicles. Thus short range based V2V communication is applicable. However, other communication factors such as handshake time of communication, throughput, response time, etc. can significantly impact V2V communication as well. Therefore, the selection of wireless technology really depends on the services and applications that the $\mathrm{V} 2 \mathrm{~V}$ communication is served.

In the future work, we plan to compare the proposed model with other models that are comparable. In addition, we will apply the model to develop a more reliable routing algorithm.

\section{REFERENCES}

[1] "National Highway Traffic Safety Administration 2012 report, (Accessed on February 5, 2013): http://www.nhtsa.gov/staticfiles/ administration/pdf/Budgets/FY2012_Budget_Overview-v3.pdf."

[2] "The US NHTSA Final Regulatory Evaluation - Office of Regulatory Analysis and Evaluation National Center for Statistics and Analysis, 2006 (Accessed on February 14, 2013). URL: http://www.nhtsa.gov/DOT/NHTSA/Rulemaking/Rules/Associated Files/EDRFRIA.pdf."

[3] H. Hartenstein and K. Laberteaux, VANET: vehicular applications and inter-networking technologies. Wiley Online Library, 2010.

[4] A. Pierce, "' crash survival systems + car-to-car communication = a next gen crash avoidance system"," in Technology Today, 2011, pp. 8-9.

[5] "Standard Specification for Telecommunications and Information Exchange between Roadside and Vehicle Systems5 GHz Band Dedicated Short Range Communications (DSRC) Medium Access Control (MAC) and Physical Layer (PHY) Specifications (Accessed on February 10, 2013). URL: http://www.astm.org/Standards/E2213.htm.” 
[6] "Dedicated Short Range Communications

(DSRC).

(Accessed

on

6, 2013)

URL:

http://www.its.dot.gov/factsheets/dsrc_factsheet.htm.'

[7] G. Yan, B. B. Bista, D. B. Rawat, and E. F. Shaner, "General active position detectors protect vanet security," in Broadband and Wireless Computing, Communication and Applications (BWCCA), 2011 International Conference on, 2011, pp. 11-17.

[8] D. B. Rawat, G. Yan, B. B. Bista, and M. C. Weigle, "Trust on the security of wireless vehicular ad-hoc networking," Ad Hoc \& Sensor Wireless Networks (AHSWN) Journal, to appear.

[9] D. B. Rawat, B. B. Bista, G. Yan, and M. C. Weigle, "Securing vehicular ad-hoc networks against malicious drivers: a probabilistic approach," in Complex, Intelligent and Software Intensive Systems (CISIS), 2011 International Conference on, 2011, pp. 146-151.

[10] J. A. Fernandez, K. Borries, L. Cheng, B. V. Kumar, D. D. Stancil, and F. Bai, "Performance of the $802.11 \mathrm{p}$ physical layer in vehicle-to-vehicle environments," Vehicular Technology, IEEE Transactions on, vol. 61, no. 1, pp. 3-14, 2012.

[11] R. C. Daniels and R. W. Heath, "Link adaptation with position/motion information in vehicle-to-vehicle networks," Wireless Communications, IEEE Transactions on, vol. 11, no. 2, pp. 505-509, 2012.

[12] M. Abuelela, S. Olariu, and M. C. Weigle, "NOTICE: An architecture for the notification of traffic incidents," in Vehicular technology conference, 2008. VTC Spring 2008. IEEE, 2008, pp. 3001-3005.

[13] D. B. Rawat, D. C. Popescu, G. Yan, and S. Olariu, "Enhancing vanet performance by joint adaptation of transmission power and contention window size," Parallel and Distributed Systems, IEEE Transactions on, vol. 22, no. 9, pp. 1528-1535, 2011.

[14] M. Karpiriski, A. Senart, and V. Cahill, "Sensor networks for smart roads," in Pervasive Computing and Communications Workshops, 2006. PerCom Workshops 2006. Fourth Annual IEEE International Conference on, 2006, pp. 5-pp.

[15] D. Rawat, D. Treeumnuk, D. Popescu, M. Abuelela, and S. Olariu, "Challenges and perspectives in the implementation of notice architecture for vehicular communications," in Mobile Ad Hoc and Sensor Systems, 2008. MASS 2008. 5th IEEE International Conference on, 2008, pp. 707-711.

[16] S. Panichpapiboon, G. Ferrari, and O. K. Tonguz, "Connectivity of ad hoc wireless networks: an alternative to graph-theoretic approaches," Wirel. Netw., vol. 16, no. 3, pp. 793-811, 2010.

[17] M. Kiese, C. Hartmann, J. Lamberty, and R. Vilzmann, "On connectivity limits in ad hoc networks with beamforming antennas," EURASIP J. Wirel. Commun. Netw., vol. 2009, pp. 1-1, 2009.

[18] Q. Yang, A. Lim, S. Li, J. Fang, and P. Agrawal, "ACAR: Adaptive connectivity aware routing for vehicular ad hoc networks in city scenarios," Mob. Netw. Appl., vol. 15, no. 1, pp. 36-60, 2010.

[19] A. Kesting, M. Treiber, and D. Helbing, "Connectivity statistics of store-and-forward intervehicle communication," IEEE Transactions on Intelligent Transportation Systems, vol. 11, no. 1, pp. 172-181, 2010.

[20] F. Bai, N. Sadagopan, B. Krishnamachari, and A. Helmy, "Modeling path duration distributions in manets and their impact on reactive routing protocols," IEEE Journal on Selected Areas in Communications, vol. 22, no. 7, pp. 1357-1373, Sept. 2004.

[21] I. Gruber and L. Hui, "Link expiration times in mobile ad hoc networks," Nov. 2002, pp. 743-750.

[22] M. Pascoe-Chalke, J. Gomez, V. Rangel, and M. Lopez-Guerrero, "Route duration modeling for mobile ad-hoc networks," Wirel. Netw., vol. 16, no. 3, pp. 743-757, 2010.

[23] M. Nekovee, "Modeling the spread of worm epidemics in vehicular ad hoc networks," in Proceedings of the 63-th IEEE Vehicular Technology Conference VTC Spring 2006, Melbourne, Australia, 7-10 May 2006, pp. 841-845.

[24] M. Nekovee and M. Ko, "Throughput analysis of Wi-Fi based broadband access for mobile users on the highway," in Proceedings of 13-th IEEE International Conference on Networks, Boston, Massachusetts, USA, 16-18 Nov 2005, p. 6.

[25] W. Sun, H. Yamaguchi, and K. Yukimasa, "GVGrid: A qos routing protocol for vehicular ad hoc networks," in Proceedings of the Fourteenth IEEE International Workshop on Quality of Service (IWQoS 2006), Yale University, New Haven, CT, USA, June 19-21 2006, pp. 130-139.

[26] T. S. Rappaport, Wireless communications principles and practices. Prentice-Hall, 2002.

[27] A. Visser, H. Yakali, A.-J. van der Wees, M. Oud, G. van der Spek, and L. Hertzberger, "A hierarchical view on modeling the reliability of a DSRC link for ETC applications," IEEE Transactions on Intelligent Transportation Systems, vol. 3, no. 2, pp. 120 - $129,2002$.

[28] G. Yan and S. Olariu, "A probabilistic analysis of link duration in vehicular ad hoc networks," IEEE Transactions on Intelligent Transportation Systems, vol. 12, no. 4, pp. 1227-1236, 2011.

[29] T. Rappaport, Wireless Communications: Principles and Practice, 2nd ed. Upper Saddle River, NJ, USA: Prentice Hall PTR, 2001.

[30] N. Beaulieu and Q. Xie, "An optimal log-normal approximation to log-normal sum distributions," IEEE Transactions on Vehicular Technology, vol. 53, no. 2, pp. 479-489, March 2004.

[31] Z. Liu, J. Almhana, and R. McGorman, "Approximating log-normal sum distributions with power log-normal distribution," IEEE Transactions on Vehicular Technology, vol. 43, no. 4, pp. 2611-2617, July 2008.

[32] L. Fenton, "The sum of log-normal probability distributions in scatter transmission systems," IEEE Transactions on Communications, vol. 8, no. 1, pp. 57-67, 1960.

[33] R. Krasteva, A. Boneva, G. Vesselin, and I. Stoianov, "Application of Wireless Protocols Bluetooth and ZigBee in Telemetry System Development," Problems of Engineering, Cybernetics, and Robotics, vol. 55, pp. 30-38, 2005.

[34] R. Troutbeck and W. Brilon, Unsignalized Intersection Theory, 1997. 